\begin{tabular}{lllllllllllllllllllllllllllll}
\hline $\mathrm{A}$ & $\mathrm{C}$ & $\mathrm{T}$ & $\mathrm{A}$ & $\mathrm{A}$ & $\mathrm{R}$ & $\mathrm{C}$ & $\mathrm{H}$ & $\mathrm{A}$ & $\mathrm{E}$ & $\mathrm{O}$ & $\mathrm{L}$ & $\mathrm{O}$ & $\mathrm{G}$ & $\mathrm{I}$ & $\mathrm{C}$ & $\mathrm{A}$ & & $\mathrm{C}$ & $\mathrm{A}$ & $\mathrm{R}$ & $\mathrm{P}$ & $\mathrm{A}$ & $\mathrm{T}$ & $\mathrm{H}$ & $\mathrm{I}$ & $\mathrm{C}$ & $\mathrm{A}$
\end{tabular}

VOL. LIV (2019): 191-206

PL ISSN 0001-5229

DOI 10.4467/00015229AAC.19.010.11887

\author{
WoJCIECH NowaKowski
}

\title{
Ein VOLK IM Schatten SEINER NACHBARN - die Buren und die Przeworsk-Kultur
}

\section{A PEOPLE IN THE SHADOW OF THEIR NEIGHBOURS - Buri ANd The Przeworsk culture}

Zusammenfassung: Tacitus erwähnte in seiner Germania das Volk der Buri, dessen Sitz sich in der Nähe der Karpaten oder genau genommen in diesen Bergen befinden sollte, was auf dessen Nachbarschaft mit der lugischen Föderation (Lugiorum nomen) hinweist, die allgemein als die Bevölkerung der Przeworsk-Kultur identifiziert wurde. Ptolemäus schrieb hingegen über die Lugioi Buroi, was eine Zugehörigkeit dieses Stammes zum Lugierbund andeutete. Man kann vermuten, dass die Volksnamen Buri und Lugioi Buroi denselben Stamm bezeichneten, der einen Teil der Bevölkerung sowohl der Przeworsk-Kultur, als auch des lugischen Völkerverbandes darstellte. Ihr Sitz lag in der älteren Kaiserzeit in der von keltischen und dakischen Einflüssen geprägten „Südzone“ der Przeworsk-Kultur in Südostpolen. Die Buren, wie auch die gesamte lugischen Föderation, waren südlich der Karpaten politisch und militärisch aktiv, unter anderen während der Dakerkriege Trajans, und drangen in der zweiten Hälfte des 2. Jh.s in das Karpatenbecken ein, um dessen Nordrand an der Obertheiß zu besiedeln. Die Grenzlage der Buren in Kleinpolen, am Südrand des lugischen Territoriums, hatte zur Folge, dass die Römer sie sowohl als Mitglied der Föderation (Lugioi Buroi), als auch als „separates“ Volk (Buri) wahrnahmen.

Sprachkorrektur von Martin Lemke

Stichworte: Buri; Lugiorum nomen, Barbaricum, römische Kaiserzeit, Germania von Tacitus, Geographia von Ptolemäus

Abstract: In his Germania, Tacitus mentions the people of Buri, whose settlements were located near or in the Carpathian Mountains, which would indicate that they were neighbors with the Lugian federation (Lugiorum nomen), commonly identified with the Przeworsk culture population. In turn, Ptolemy wrote about Lugioi Buroi, which would suggest that this tribe was a part of the Lugian federation. It can be assumed that the ethnonyms Buri and Lugioi Buroi referred to the same people, who were both a part of the Przeworsk culture population and a branch of the Lugian

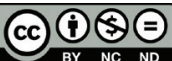

This is an open access article distributed under the terms of the Creative Commons license (CC BY-NC-ND 3.0 PL) 
federation. The settlements of this tribe in the Early Roman Period were located in the southern part of the Przeworsk culture territory, in southeastern Poland, in an area that at that time was under strong Celtic and Dacian influences. The Buri, like the entire Lugian federation, were politically and militarily active in the lands south of the Carpathians, for example, during Trajan's Dacian Wars. In the second half of the 2nd century AD, they invaded the Carpathian Basin, settling its northern edge over the Upper Tisa. The border location of the Buri, on the southern outskirts of the Lugian territory, caused them to be perceived by the Romans as both a part of the Lugian federation (Lugioi Buroi) and as a "separate" people (Buri).

translated by Kinga Brzezińska

Keywords: Buri, Lugiorum nomen, Barbaricum, Roman Period, Geography of Ptolemy, Germania of Tacitus

Dem großen polnischen Gelehrten Jerzy Kolendo zufolge bildete die Germania von Tacitus die umfangreichste ethnographische Beschreibung des mitteleuropäischen Barbaricums in der römischen Kaiserzeit (Kolendo 2008a: 19-24; vgl. Lund 1988, 44-69). Der für die polnische Archäologie wichtigste Teil dieses Werkes befindet sich zwischen den beiden berühmten Sätzen „Nun ist über die Sueben zu sprechen" (Nunc de Suebis dicendum est - TAC., Germ., 38,1; vgl. Lund 1988, 99) und „Hier endet das Land der Sueben“ (Hic Suebiae finis - TAC., Germ., 45,6; vgl. Lund 1988, 107). Der Abschnitt stammt höchstwahrscheinlich aus einem Bericht des römischen diplomatischen Dienstes, der nach dem Besuch des Königs der Semnonnen, Masyos in der letzten Dekade des 1. Jh.s n.Chr. im römischen Kaiserreich entstand (Kolendo 2007, 230-237; 2008a, 35-44). Dieses hypothetische „Descriptio Suebiae“ bildete gleichzeitig die älteste schriftliche Quelle für die Geschichte der Völker, die im Oder-, Warthe- und Weichselgebiet wohnten, also in einem Raum, der sich etwa mit dem Territorium heutigen Polens deckt (Kolendo 2008a, 44-46).

Dieses Gebiet wurde nach Tacitus größtenteils von der lugischen Föderation bewohnt: Mit Beschreibungen wie „der Stammesverband der Lugier dehnt sich am weitesten aus" (Lugiorum nomen latissimae patet - TAC., Germ., 43,2; vgl. Lund 1988, 103; Kolendo 2008b, 160-162) betonte Tacitus die Größe dieses Bundes. Von den zu dieser Föderation gehörenden Stämmen nannte er lediglich fünf Völker - die Harier, Helvekonen, Manimer, Helisier und Nahanarvaler - welche als die stärksten galten (valentissimas nominasse suffieciet: Harios, Helveconas, Manimos, Helisios, Nahanarvalos - TAC., Germ., 43,2; vgl. Lund 1988, 103). Ferner beschrieb Tacitus einen im Territorium der Nahanarvaler liegenden heiligen Hain, der eine gemeinsame Kultstelle für den gesamten Bund war (Timpe 1992, 467-743; Tausend 1997, 233). Die ziemlich detaillierte Darstellung 
der Rituale und die exponierte Rolle dieses religiösen Zentrums führte einige Historiker zum Schluss, dass der Lugiorum nomen vor allem ein Kultverband war (Schmidt 1942, 3; Tausend 1997, 230-231). Wesentlich öfter wurde aber auf den Schutz der Bernsteinstraße als Grund für das Entstehen dieses Bündnisses hingewiesen (Łowmiański 1963, 229). Es ist leider nicht sicher, ob die weiter im Text folgende Beschreibung von Kriegern mit schwarz bemalten Schilden, die infolge ihrer Wildheit und nächtlichen Kampfweise ein "Gespensterheer" bildeten (feralis exercitus - TAC., Germ., 43,4; vgl. Lund 1988, 103), sich nur auf die Harier, einen anderen der „wichtigsten Stämme“ des Lugierbunds bezieht, oder ob diese die gesamte Völkergemeinschaft betrifft (Lund 1989, 226-227; Timpe 1992, 469-470). Sollte letzteres der Fall sein, so ist anzunehmen, dass es sich um eine Art „Bündnisarmee“ handeln konnte (vgl. Kolendo 2005, 111; 2008b, 165-169; 2012).

Eine wesentlich geringere Aufmerksamkeit schenkte Tacitus den Völkern, die er selbst oder seine Informanten, als nicht so bedeutend ansahen wie die „Wächter“ der Bernsteinstraße. Dies betrifft, unter anderen, die vier Völker der Marsinger, Kotiner, Osen und Buren (Marsingi, Cotini, Osi, Buri - TAC., Germ., 43,1), die in einem Landstreifen zwischen den Markomannen und Quaden - beides wichtige Partner der Römer - einerseits und eben den Lugiern, ansässig sein sollten (vgl. Kolendo 1999, 222; vgl. auch Godłowski 1963, 100). Die Wohnsitze dieser Stämme befanden sich größtenteils „auf bewaldeten Höhen, Berggipfeln und auf einer Bergkette"(omnesque hi populi pauca campestrium, ceterum saltus et vertices montium iugumque insederunt - TAC., Germ., 43,1-2; vgl. Lund 1988, 103), was auf ein Gebiet in der Nähe oder in den Karpaten selbst hinweist (Kolendo 2004, 19-20). Den zuletzt in dieser Völkerkette erwähnten, also am weitesten im Osten befindlichen Buren wurde nur eine zusätzliche Bemerkung gewidmet: sie sollten nämlich „den Sueben in Sprache und Lebensweise“ ähneln (Buri sermone cultuque Suebos referunt-TAC., Germ., 43,1; vgl. Lund 1988, 103), wodurch sie sich deutlich von ihren Nachbarn - den gallischsprachigen Kotinern und den pannonischen Osen - unterscheiden.

Eben die Nachbarschaft mit diesen beiden Völkern war für Jerzy Kolendo ein Grund, den Sitz der Buren innerhalb des Karpatenbeckens, an dessen Nordrand, im oberen Theißgebiet, zu verorten (Kolendo 1999, 221-225; 2008b, 159-160). Diese Deutung scheinen einige Überlieferungen zu stützen, die über eine große Aktivität der Buren und ihre Kontakte und Konflikte mit den Römern während der dakischen und markomannischen Kriege berichten (Dio, Hist. Rom., LXVIII, 8,1-2; LXXI (LXXII) 18,1; LXXII (LXIII) 2,4; SHA, Vita Marci, 22,1; vgl. Wenskus 1961, 457; Strobel 1984, 175, 183-184; Stahl 1989, 301). Ein Friedenschluss mit dem Kaiser Kommodus, dem zufolge die Buren einen 40 römischen Meilen breiten Streifen in der Nähe der Grenze Dakiens räumen sollten, bestätigt die Lage ihrer Heimat im Karpatenbecken. 
Ein Volksname, der den beiden von Tacitus erwähnten Ethnonymen ähnelt, kommt in der Geographia von Ptolemäus vor, der drei lugische Stämme nannte.

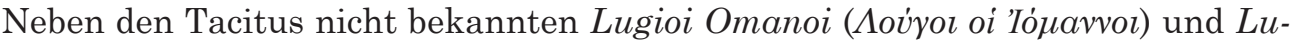

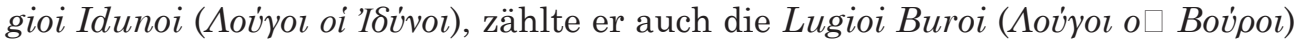
dazu (PтоL., Geogr. II, 11, 10; vgl. Cuntz 1923, 64-65). Das letztere Volk wurde oft als mit den Buren bei Tacitus identisch angesehen (Steche 1937, 63), obwohl Ptolemäus dessen Sitz beim Askiburgion-Gebirge und in der Nähe der Weichselquellen verortete, was zur Folge hat, dass manche Forscher die Heimat dieses Stammes in Schlesien (vgl. Ahlquist 1972) oder Mähren (vgl. Steche 1937, 60, 146, 149; Wenskus 1977, Karte 1; BöHme 1975, Abb. 22) suchten, also weit westlich vom oben vorgeschlagenen Land der Buren. Dieser Unterschied der Lokalisierung wurde gleichzeitig zum Anlass genommen, die Lugioi Buroi und die Buren als die zwei unterschiedlichen Völker zu betrachteten (Olędzki 2008, 158-161; 2017).

Ein weiteres Problem stellt die Zugehörigkeit der Buren zum Lugierbund dar. Die Unterschiede in den Überlieferungen von Tacitus und Ptolemäus werden mit einem Wechsel der politischen Lage begründet, basierend auf zwei Möglichkeiten: entweder schlossen die „suebischen“ Buren im 2. Jahrhundert n.Chr., also nach der Entstehen des Werkes von Tacitus, ein Bündnis mit den Lugiern ab (Steche 1937, 63), oder aber sie waren umgekehrt schon in der Zeit von Tacitus aus dem lugischen Bund ausgetreten, so dass Ptolemäus eine nicht mehr aktuelle Situation beschrieben hat (Haider 2008, 29). Andererseits wurden jedoch die Buren von Tacitus mitunter, eben aufgrund der Ptolemäischen Überlieferung, einfach dem lugischen Bund zugeschrieben (vgl. Schmidt 1910, 354; 1942, 5; Courtois 1955, 24).

Diese verwickelte Problematik versuchte Jerzy Kolendo vor schon fast zwanzig Jahren zu ordnen, als er eine neue Lösung für die Buren-Problematik vorschlug. Er betonte, dass für die antike Geographie die Lage der Quelle oder auch nur des Oberlaufs eines sogar großen Flusses, immer ein riesiges Problem darstellte. Das beste Beispiel ist die Elbe, bei der die Moldau als ihr Oberlauf angesehen wurde (Kolendo 1998, 128, Anm. 45). Nicht selten wurden einem Fluss zwei Quellen zugeschrieben, was, der Überlieferung von Ptolemäus nach (Ртоц., Geogr., II, 11, 2; vgl. Cuntz 1923, 60-61), auch die Weichsel zu betreffen scheint, welche zwei verschiedenen und ziemlich weit voneinander entfernte Quellen haben sollte. Eine davon befand sich im Westen, nördlich der Mährischen Pforte. Neben der tatsächlichen Quelle könnte aber auch der Oberlauf der Oder für die antike Sichtweise in Frage kommen. Die zweite der von Ptolemäus erwähnten Weichselquellen sollte weit im Osten liegen und lässt sich daher mit großer Wahrscheinlichkeit mit dem Oberlauf des San identifizieren. Diese „östliche Weichselquelle“ voraussetzend lassen sich die Lugioi Buroi in diesem Gebiet verorten, das durchaus in der Nähe der Heimat der Buren bei Tacitus lag (Kolendo 1999, 218-221, 226). 
Jerzy Kolendo war der Meinung, dass sich beide Namen - Buri und Lugioi Buroi - auf dasselbe Volk bezogen, das am Nordrand des Karpatenbeckens lebte (Kolendo 1999). Die Ptolemäische Version des Ethnonyms dürfte man als eine Spur des Eintritts der Buren in den Lugierbund deuten, welcher vermutlich im frühen 2. Jh. vollzogen wurde, in der Zeit der größten Entwicklung der Bernsteinstraße und gleichzeitig des Apogäums des davon profitierten Stammesverbandes. Eine archäologisch erfassbare Konsequenz dieser Ereignisse war die Einwanderung der „lugischen“ Bevölkerung der Przeworsk-Kultur ins Obertheißgebiet- diese Migration soll eben durch das Bündnis mit den Buren möglich gewesen sein (Kolendo 1999, 227-228).

Diese Hypothese hat jedoch zwei Schwachstellen. Erstens nutzte Ptolemäus in seiner Geographie (wie Jerzy Kolendo selbst vielfach betonte - z.B. Kolendo 1998, 110-111; Kolendo, Płóciennik 2015, 230-231), ältere Texte, vor allem das verlorene, einige Dekaden ältere Buch des Marinos von Thyros (Polaschek 1965, col. 695-696, 753-757; Reichert 2003, 569). Man sollte also seine Beschreibung des mitteleuropäischen Barbaricums als der Germania von Tacitus zeitgenössisch betrachten. Beide Autoren beschreiben die Situation in der zweiten Hälfte des 1. Jh.s. Die Unterschiede zwischen den beiden Überlieferungen lassen sich also nicht chronologisch erklären.

Zweitens wies Jerzy Kolendo auf keinerlei archäologische Funde aus dem Nordrand des Karpatenbeckens hin, die als Hinterlassenschaft der Buren zu deuten wären. Bis zur Mitte des 2. Jh.s kamen in dieser Region Fundstellen vor, die sich mit den dakischen Kulturen verbinden lassen (vgl. Budinský-Krička 1963, 33; Lamiová-Schmiedlová 1969, 458-461, Abb. 37; Budinský-Krička, Lamiová-Schmiedlová 1990, 313-314; Madyda-Legutko 1996, 45-47), was überhaupt nicht zu dem von Tacitus ausdrücklich betonten „suebischen“ Kulturmodell der Burenm passt.

Es scheint, dass man von der Feststellungen von Jerzy Kolendo ausgehend und die Ergebnisse seiner Analyse der Schriftquellen durchaus wertschätzend, eine neue, tiefer greifende Hypothese formulieren kann, die wesentlich mehr auf die archäologischen Funde eingeht.

Der Ausgangspunkt ist die grosso modo akzeptierte Kongruenz der Lugier mit der Bevölkerung der Przeworsk-Kultur (vgl. Godłowski 1985, 140-143; 2000, 251-254; Kolendo 2005, 112-118). Diese seit der jüngeren vorrömischen Eisenzeit existierende archäologische Formation (vgl. KokowsKi 2003) scheint in der älteren Kaiserzeit ziemlich homogen gewesen zu sein. Innerhalb ihres Territoriums kann man keine deutlichen Siedlungskonzentrationen aussondern, die eine eigene kulturelle „Färbung“ zeigen würden. Allerdings lässt sich in ihrem östlichen Gebiet eine lokale Version der Przeworsk-Kultur registrieren. Diese so genannte östliche Zone (Andrzejowski 2001) hat keine scharfe Grenze, und lässt sich auch weder mit einem der lugischen Stamme, noch mit irgendeinem konkreten anderen barbarischen Volk identifizieren. 


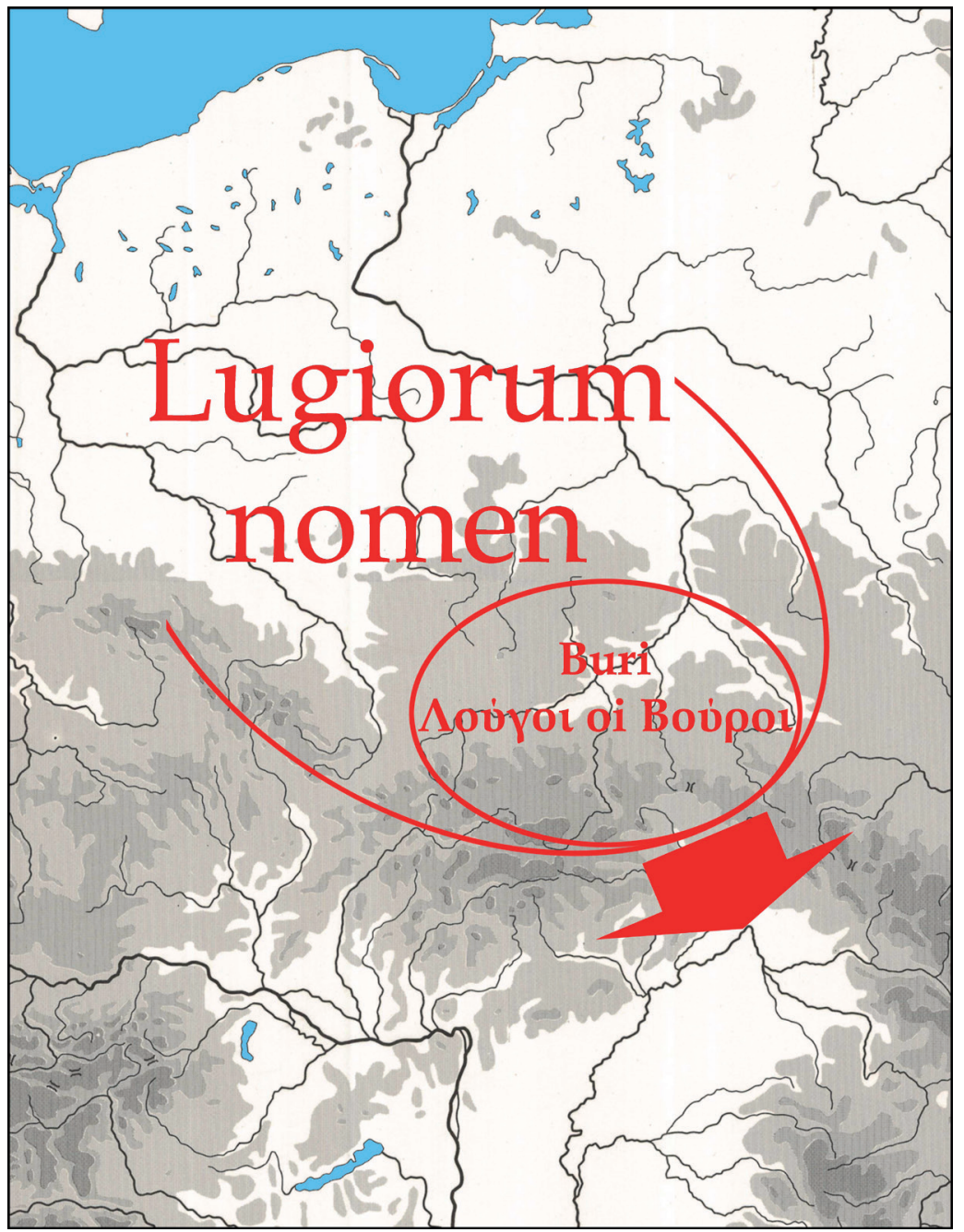

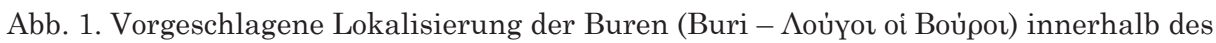
lugischen Bundes (Lugiorum nomen) und die Richtung ihr Expansion in den Karpatenbecken

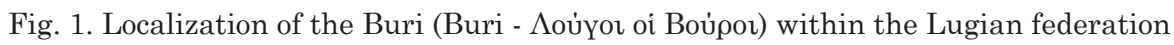

(Lugiorum nomen) and the direction of their expansion in the Carpathian Basin

Die Ergebnisse der in den letzten zwanzig Jahren durchgeführten Ausgrabungen und analytischen Studien gestatten es, eine weitere „lokale Variante“ der Przeworsk-Kultur in Kleinpolen aufzuzeigen. Das kulturelle Antlitz dieses Raumes wurde in der jüngeren vorrömischen Eisenzeit stark von den 
Einflüssen der Latène-Kultur geprägt, die sich auch als Spuren der Anwesenheit der ethnischen Kelten deuten lassen (Woźniak 1990, 73-79; 2004; Poleska 1996; 2005; 2006; Madyda-Legutko 2004, 71-72; Karwowski 2007; 2008). Auch später, in der älteren römischen Kaiserzeit, kommen in dieser Region Fundstellen der Púchov-Kultur vor (Madyda-Legutko 2004, 73), deren Bevölkerung als die „gallischsprachigen“ Kotiner anzusehen sind (Kolendo 2004, 18-19). Gleichzeitig treten im Südostteil dieses Gebiets Funde der Wietrzno-Solina-Gruppe auf, die auf Verbindungen mit der dakischen Besiedlung im Karpatenbecken hinweisen (Madyda-Legutko 1996, 51-54, 61-66; 2004, 72; vgl. Florkiewicz 2004). Die Ergebnisse der Ausgrabungen aus den letzten Jahren zeugen von einer wesentlich dichteren Besiedlung der Przeworsk-Kultur in der älteren Kaiserzeit, als bisher angenommen, die sich auch weiter nach Süden ins Karpatenvorland ausdehnte (Madyda-Legutko 2004, 73-79, Abb. 1-2; Florkiewicz, Reszczyńska 2007, 148-153; vgl. Madyda-Legutko, Pohorska-Kleja, Rodzińska-Nowak 2009; Madyda-Legutko, Rodzińska-Nowak, Zagórska-Telega 2009). Die dortigen Funde ähneln offenbar auch den Materialien aus der östlichen Zone (Madyda-Legutko, Rodzińska-Nowak, Zagórska-Telega 2009, 301; Madyda-Legutko, RodzińskaNowak 2009, 48), was wohl als Spur einer „Nebenroute“ der Bernsteinstraße anzusehen ist: Diese verlief aus dem Karpatenbecken über Gebirgspässe in den Beskiden und dann den San und Mittelweichsel entlang weiter nach Norden, über Nordmasowien und Masuren zur Bernsteinküste (Kolendo 1999, 219; vgl. Nowakowski 2017, 127-128). Alle diese Eigenschaften betonen den Unterschied zwischen der „Südzone“ im Oberweichsel- und Sangebiet und dem „orthodoxen“ Modell der Przeworsk-Kultur in Schlesien und Großpolen.

Als Illustration der Eigenart der hypothetischen „Südzone“ der PrzeworskKultur können zwei dort entdeckte reiche Gräber dienen. In Giebułtów wurde eine Brandbestattung gefunden, mit einer Beigabe, die neben einer „fürstlichen“ Goldkette unter anderem auch zwei auf der Drehscheibe gefertigte Fußbecher und einen ebenso hergestellten Krug umfasste (Nosek 1961; Dobrzańska, Wielowiejski 1997), die der dakischen Keramik ähneln (vgl. Berciu 1981, Taf. 54). Stark verschmolzene Fragmente solcher Fußbecher stammen auch aus dem reichen Brandgrab in Sandomierz-Krakówka, wo diese mit römischen Bronzegefäßen, aber auch mit den Waffen vergesellschaftet waren (Kokowski, Ścibior 1990). Beide Elitengräber, die sich so stark von den Przeworsk-Funden des Lubieszewo Typs mit ihren Körperbestattungen unterscheiden, lagen jeweils an einem Rand der "Südzone“ und gleichzeitig in der Nähe einer der zwei in der Ptolemäischen Geographie erwähnten Quellen der Weichsel, bei denen die Heimat der Lugioi-Buroi liegen sollte. Aufgrund der dargestellten Besonderheiten scheint es legitim, sowohl die Buri, als auch die Lugioi-Buroi als ein und dasselbe Volk zu erkennen, dessen Sitz sich bei den beiden dem Ptolemäus bekannten Weichselquellen, zwischen der Oberweichsel und San, befand. 
Die Lage ihrer Heimat nördlich den Karpaten war für die Buren sicher kein Hindernis bei ihrer Aktivität im Karpatenbecken und an der unteren Donau: die Lugier (die Bevölkerung der Przeworsk-Kultur aus dem mittleren Oder-, Wartheund mittleren Weichselgebiet) griffen im 1. Jh. die Quaden an der mittleren Donau an (TAC., Ann., XII, 29 - vgl. Kolendo 1999, 227), verwickelten sich in Kämpfe an der Grenze der Provinz Moesien und erhielten militärische Unterstützung von Kaiser Domitian (Dio, HR, LXVII, 12, 5(2) - vgl. Kolendo 1999, 227).

Andererseits kann man bemerken, dass Rom die Buren wesentlich anders behandelte, als die übrigen Barbaren an der Donau. Von dieser Sonderstellung der Buren zeugt eine Mitteilung über eine burische Botschaft an Trajan, die ihm auf einem beschriebenen großen Pilz übermittelt wurde (DIO, HR, LXVIII, 8, 1-2 - vgl. Vulpe 1976, 199-200; Strobel 1984, 175, 183-184; Kolendo 1999, 223). Diese phantastische Geschichte, der zufolge, die Buren zivilisiert genug gewesen wären, um ein Schreiben, zumal mit „lateinischen Buchstaben“, verfassen zu können, aber gleichzeitig nur einen Pilz als Schriftträger zu haben, scheint eine Darstellung von exotischen Barbaren gewesen zu sein. Das angebliche burische Ultimatum, das von einem vom Pferd stürzenden „Botschafter“ übergeben wurde, war also keine reale Drohung, sondern eine komische Anekdote, die sich für die kaiserliche Propaganda, unter anderen durch eine Abbildung auf dem Triumphsäule, ausnutzen ließ (Cichorius 1896, 50-55, Taf. X:25-26; vgl. Gauer 1977, 24, Taf. 10b; Lepper, Frere 1988, 24, Taf. X). Somit wurden die Buren nicht als direkte Nachbarn und potentielle Gegner, sondern als märchenhaftes, ziemlich weit entferntes, wohl schon außerhalb des den Römern gut bekannten Karpatenbeckens wohnendes Volk dargestellt.

Ganz anders sah die Sachlage während der markomannischen Kriege aus. Eine lange, in der Historiae Augustae zitierte Liste der damals die römischen Provinzen angreifenden Barbaren (gentes omnes ab Illyrici limite usque in Galliam conspiraverant, ut Marcomanni, Varistae, Hermunduri et Quadi, Suevi, Sarmat<a>e, Lacringes et Burei † hi aliique cum Victualis, Sosibes, Sicobotes, Roxolani, Basternae, Halani, Peucini, Costoboci - SHA, Vita Marci, 22, 1; vgl. Kolendo 1999, 224), zu denen auch die Buren gehören sollen, scheint zwar eher eine Zusammenstellung aller bekannten Volksnamen gewesen zu sein (Burian 1987, 117; Birley 2010, 45), bestätigt wird jedoch der bedeutende burische Anteil in diesen Kämpfen, durch eine Information über zahlreiche, von ihnen entführte römische Gefangene, die erst nach dem Friedenschluss befreit wurden (Dio, HR, LXXII, 3,2-3). Dem Vertrag gemäß mussten die von den Römern besiegten Buren einen Grenzstreifen räumen. Mindestens ein Teil des von ihnen besetzten Gebiets lag also damals im Karpatenbecken, in der Nähe der römischen Sicherheitszone (vgl. Kolendo 1999, 225).

In derselben Zeit veränderte sich das archäologische Bild dieser Region: eine Expansion der Przeworsk-Kultur nach Süden erreichte schon das Obertheißgebiet (Godłowski 1982; 1984; 1994), wovon sowohl Kriegergräber mit Waffenbei- 
gabe (vgl. Budinský-Krička, Lamiová-Schmiedlová 1990, 249, 251-253, 314, Taf. II-III, VI-IX; Kobal' 1997, 32-38, 43, Abb. 1:d-e, 2-4, 6, 12) als auch Funde von Przeworsk-Keramik in Siedlungen (Budinský-Krička 1963, 22-24, Abb. 14-15; Lamiová-Schmiedlová 1969, 463-466; Kobal' 1997, 49) zeugen. Man kann diese Erscheinung als eine Einwanderung, wohl unter dem Druck der superiores barbari (SHA, Vita Marci, 14, 1; vgl. Godłowski 1984) erkennen, der in den antiken Quellen unter verschiedenen Namen notierten Gruppierungen der Bevölkerung der Przeworsk-Kultur, zu denen neben den erstmals erwähnten Hasdingern (Courtois 1955, 30, Karte; vgl. Wenskus 1977, 504), Lakringern und Wiktowalern (Schmidt 1941, 103) eben auch die Buren aus der „Przeworsk-Südzone“ in Kleinpolen gehören konnten. Die Buren scheinen in dieser Zeit zu einem gefährlichen Gegner der Römer geworden zu sein, von dessen Raubzügen man sich durch einen breiten Streifen „der wechselseitigen Furcht" (mutuo metu - TAC., Germ., 1; vgl. Lund 1988, 71) trennen wollte, wovon der zitierte Friedensvertrag mit Commodus zeugt. Gleichzeitig weisen die waffenführenden Gräber der PrzeworskKultur im Obertheißgebiet eindeutig darauf hin, dass die neue Bevölkerung, die dort mehr oder weniger zum Zeitpunkt der markomannischen Kriege angekommen war, keine friedliche Nachbarschaft bildete.

Man kann also annehmen, das die Volksnamen Buri und Lugioi Buroi denselben Stamm bezeichneten, der einen Teil der Bevölkerung sowohl der Przeworsk-Kultur, als auch des lugischen Völkerverbandes darstellte. Ihr Sitz lag in der älteren Kaiserzeit in der von keltischen und dakischen Einflüssen geprägten „Südzone“ der Przeworsk-Kultur in Kleinpolen, zwischen den „beiden Weichselquellen“. Die Buren, wie auch die gesamte lugischen Föderation, waren südlich der Karpaten politisch und militärisch aktiv, unter anderen während der dakischen Kriege von Trajan, und drangen in der zweiten Hälfte des 2. Jh.s in das Karpatenbecken ein, um dessen Nordrand an der Obertheiß zu besiedeln.

Die Grenzlage der Buren in Kleinpolen, am Südrand des lugischen Territoriums, hatte zur Folge, dass die Römer sie sowohl als Mitglied der Föderation, als auch ein „separates“ Volk wahrnahmen. An dieser Stelle sollte an eine schon rund 60 Jahre alte Hypothese von Max Vasmer erinnert werden, der vorschlug, den Namen Lugii als „Eidgenossen“ zu übersetzen (Vasmer 1958, 190), während das Ethnonym Buri so viel wie „Söhne“ oder „Jünglinge“ bedeuten sollte (Vasmer 1958, 193). Die beiden Volksnamen könnten also die Position der Buren, als ,jüngeres“ oder „neues“ Mitglied im Lugierbund unterstreichen. Diese attraktive Deutung scheint aber ziemlich schwach belegt zu sein.

Den Vorbehalten zum Trotz kann man noch eine andere Erklärung für die Verhältnisse zwischen den Lugiern und den Buren vorschlagen und zwar analog zur Situation im Fall der Sueben und Quaden. Die letzteren, ein suebischer Stamm mit seiner eigenen politischen Struktur und mächtigen Königen, wurden oft mit dem „allgemeinen“ Ethnonym der gesamten großen Völkergruppe bezeichnet 
(vgl. Kolendo 2005, 109), was aus der Grenzlage ihrer Heimat resultierte: der Rand des quadischen Territoriums an der Mitteldonau war gleichzeitig auch die Grenze des ganzen Suebenlandes. Ein modernes Beispiel sind heute die Slowaken und Slowenen: zwei Völker, die an den Rändern der riesigen slawischen Ökumene ansässig sind, an der Grenze mit den Ungarn beziehungsweise den Italienern, und, genau aus diesem Grund, mit lokalen Varianten des allgemeinen Ethnonyms für „Slawen“ bedacht werden.

Man kann also nicht ausschließen, dass auch die Buren, deren Sitz genauso am Rand des lugischen Gebiets lag, in den römischen Überlieferungen oft nur allgemein als „die Lugier“, ohne zusätzlichen „Einzelnamen“ bezeichnet wurden. Die vereinzelten, den „Lugiern insgesamt“ zugeschriebenen Erscheinungen, wie die berühmte „Waffenbrüderschaft“ mit den römischen Reitern in der Zeit Domitians, gehörten wohl ausschließlich zur „,separaten“ Geschichte der Buren, die für die Römer in dem im Titel dieses Vortrags genannten Schatten ihrer „großen lugischen Brüder“, nicht bemerkbar waren.

Die vorgelegte Hypothese stellt selbstverständlich keine endgültige Erklärung dar, sie zeigt lediglich die Probleme, welche jede Konfrontation vor archäologischen Funden mit antiken Überlieferungen verursacht. Antiken Schriften beschrieben das mitteleuropäische Barbaricum nach ihren Regeln, was beispielsweise in einer eigenen, oft vom heutigen Wissen abweichenden Verortung von Flussquellen oder Bergpässen resultiert. Genauso könnte ein Volk unter zwei verschiedenen Namen an zwei verschiedenen Plätzen registriert worden sein, so wie es wohl im Fall der Buri und Lugioi Buroi war. Trotz aller Schwierigkeiten gehören derartige Versuche zu den Aufgaben unserer Disziplin, die heute keine Angst davor haben sollte, dass sie in einen Forschungsbereich vorrückt, der „,zwei Zoll von dem, was man Geschichte nennt" entfernt liegt, wie Anatol France vor hundert Jahren die damaligen Archäologen verspottete (a deux doigts de ce qu'on appelle l'histoire - France 1984, 604).

\section{LITERATURVERZEICHNIS}

Ahlquist L.

1972 Notes on the Silesian Lugii, Arctos: Acta Philologica Fennica, NS 7, p. 5-12. Andrzejowski J.

2001 Wschodnia strefa kultury przeworskiej - próba definicji, Wiadomości Archeologiczne 54, 1999-2001, p. 59-87.

Berciu D.

1981 Buridava dacică, Biblioteca de arheologie 40, Bucharest.

Birley A.R.

2010 Marcus Aurelius' northern wars in the Historia Augusta, Historiae Augvstae Colloqvia, NS 11, p. 37-49. 
Böhme H.W.

1977 Archäologische Zeugnisse zur Geschichte der Markomannenkriege (166-180 n.Chr.), Jahrbuch des Römisch-Germanischen Zentralmuseums Mainz 22, p. 153-217. (1975)

Budinský-Krička V.

1963 Sidlisko $z$ doby rímskej a zo začatkov stahovania národov v Prešove, Slovenská Archeológia 11, p. 5-58.

Budinský-Krička V., Lamiová-Schmiedlová M.

1990 A late 1st century B.C. - 2nd century A.D. cemetery at Zemplín, Slovenská Archeológia 38(2), p. 245-344.

Burian J.

1987 Die Darstellung der Markomannenkriege in den SHA (Vita Marci) und ihre Glaubwürdigkeit, Listy Filologické 110, p. 114-118.

Cichorius C.

1896 Die Reliefs der Traianssäule. Zweiter Textband: Commentar zu den Reliefs des ersten dakischen Krieges, Berlin.

Courtois Chr.

1955 Les Vandales et l'Afrique, Paris.

Cuntz O.

1923 Die Geographie des Ptolemaeus. Galliae, Germania, Raetia, Noricum, Pannoniae, Illyricum, Italia, Handschriften, Text und Untersuchung, Berlin.

Dębiec M., Wołoszyn M. (eds.)

2007 Frühzeit Ostmitteleuropas: Das polnisch-ukrainische Grenzgebiet aus archäologischer Per-pektive, Collectio Archaeologica Ressoviensis, 5, Rzeszów.

Dobrzańska H., Wielowiejski J.

1997 The Early Roman high-status elite grave in Giebuttów near Cracow, Materiały Archeologiczne 30, p. 81-102.

Florkiewicz I.

2004 Problem dackich elementów kulturowych $w$ Karpatach polskich oraz na Podkarpaciu we wczesnym okresie wpływów rzymskich - stan badań, [in:] Okres lateński i rzymski w Karpatach polskich, J. Gancarski (ed.), p. 659-678.

Florkiewicz I., Reszczyńska A.

2007 Barbaricum Germanicorum - Barbaricum Europae Orientalia. Die Besiedlung zwischen der Weichsel, dem oberen Bug und dem Djestr vom 3. Jh. v.u.Z. bis zum 5. Jh. u.Z., [in:] M. Dębiec, M. Wołoszyn (eds.), p. 141-160.

France A.

1984 Oeuvres, v.I, Bibliotheque de la Pleiade, Paris (Gallimard).

Gancarski J. (ed.)

2004 Okres lateński i rzymski w Karpatach polskich, Krosno.

Godłowski K.

1963 W sprawie lokalizacji Kotynów w Górach Świętokrzyskich, Acta Archaeologica Carpathica 5(1-2), p. 91-102.

1982 Pótnocni barbarzyńcy i wojny markomańskie w świetle archeologii, [in:] Znaczenie wojen markomańskich dla państwa rzymskiego i pótnocnego Barbaricum, J. Wielowiejski (ed.), Scripta Archaeologica 2, Warszawa, p. 48-80.

1984 „Speriores barbari” und die Markomannenkriege im Lichte archäologischer Quellen, Slovenská Archeológia 32(2), p. 327-350. 
1985 Przemiany kulturowe $i$ osadnicze $w$ południowej $i$ środkowej Polsce $w$ młodszym okresie przedrzymskim $i$ w okresie rzymskim, Wrocław-Warszawa-KrakówGdańsk-Łódź.

1994 Die Barbaren nördlich der Westkarpaten und das Karpatenbecken - Einwanderungen, politische und militärische Kontakte, Specimina Nova, A Herman Ottó Múzeum Évkönyve 1993, Miskolc, p. 65-89.

2000 Pierwotne siedziby Stowian, Wybór pism pod redakcją M. Parczewskiego, Kraków. Haider P.W.

2008 „Vandalen“ in Polen: Kulturkontakt, Kulturtransfer und Ethnogenese zwischen ca. 100 v. und 200 n. Chr., [in:] Das Reich der Vandalen und seine (Vor-) Geschichten, Österreichische Akademie der Wissenschaften, PhilosophischHistorischen Klasse, Denkschriften 366 - Forschungen zur Geschichte des Mittelalters 13, G.M. Berndt, R. Steinacher (eds.), Wien, p. 17-41.

Karwowski M.

2007 Hic Celticorum Finis - Die keltische Besiedlung im polnisch-ukrainischen Grenzgebiet, [in:] frühzeit ostmitteleuropas: Das polnisch-ukrainische grenzgebiet Aus archäologischer perspective, M. Dębiec, M. Wołoszyn (eds.), p. 127-141.

2008 Neue Forschungen zur peripheren Enklave der La-Tène-Kultur im südostpolnischen Karpatenvorland, [in:] Ritus und Religion in der Eisenzeit, Beiträge zur Ur- und Frühgeschichte Mitteleuropas, 49, Ch. Eggl, P. Trebsche, I. Balzer, J. Fries-Knoblach, J.K. Koch, H. Nortmann, J. Wiethold (eds.), Langenweissbach, p. $143-154$.

Kobal' I.V.

1997 Kultura przeworska na Ukrainie Zakarpackiej, Wiadomości Archeologiczne 53(2),1993-1994, p. 31-56.

Kokowski A.

2003 Die Przeworsk-Kultur - ein Völkerband zwischen 200 vor Chr. und 375 nach Chr., [in:] Die Vandalen. Die Könige. Die Eliten. Die Krieger. Die Handwerker, Chr. Leiber, A. Kokowski (eds.), Nordstemmen, p. 77-183.

Kokowski A., Ścibior J.

1990 Tombe princière de Sandomierz-Krakówka, pèriode romaine prècoce, Inventaria Archaeologica Pologne 63, PL 385. (1993)

Kolendo J.

1998 Świat antyczny $i$ barbarzyńcy. Teksty, zabytki, refleksja nad przeszłościa I, Warszawa.

1999 Lugiowie Burowie oraz Burowie. Przyczynek do interpretacji sytuacji politycznej i kulturowej Europy barbarzyńskiej w końcu I wieku i w II wieku n.e., [in:] Comhlan. Studia $z$ archeologii okresu przedrzymskiego i rzymskiego w Europie Środkowej dedykowane Teresie Dabrowskiej w 65. rocznice urodzin, J. Andrzejowski (ed.), Warszawa, p. 217-231.

2004 Karpaty w koncepcjach geograficznych świata starożytnego, [in:] Okres lateński i rzymski w Karpatach polskich, J. Gancarski (ed.), p. 13-27.

2005 Mapa etniczna środkowoeuropejskiego Barbaricum. Swebia $i$ Lugiowie $w$ Germanii Tacyta, [in:] Archeologia o poczatkach Stowian, P. Kaczanowski, M. Parczewski (eds.), Kraków, p. 103-118.

2007 Politykagermańska Domicjana. Spotkanie cesarza z królem Semnonów Masyosem i wieszczkq Ganna, [in:] Grecy, Rzymianie $i$ ich sqsiedzi, K. Nawotka, M. Pawlak (eds.), Antiquitas 29, p. 225-239. 
2008a Wstę, [in:] P. Cornelius Tacitus, Germania-Publiusz Korneliusz Tacyt, Germania, przekład: T. Płóciennik, Wstęp i komentarz: J. Kolendo, Fontes Historiae Antiquae 10, Poznań, p. 9-57.

2008b Komentarze, [in:] P. Cornelius Tacitus, Germania-Publiusz Korneliusz Tacyt, Germania, przekład: T. Płóciennik, Wstęp i komentarz: J. Kolendo, Fontes Historiae Antique 10, Poznań, p. 105-205.

2012 Der Stamm der Harier in der Germania des Tacitus als Gefolgschaft, Eos: commentarii Societatis Philologae Polonorum 99, p. 155-165.

Kolendo J., Płóciennik T.

2015 Vistula amne discreta. Greckie i tacińskie źródła do najdawniejszych dziejów ziem Polski, Warszawa.

Lamiová-Schmiedlová M.

1969 Römerzeitliche Siedlungskeramik in der Südostslovakei, Slovenská Archeológia 17(2), p. 401-501.

Lepper F., Frere S.

1988 Trajans Column. A new edition of the Cichorius Plates. Introduction, commentary and notes, Gloucester.

Lund A.A.

1988 P. Cornelius Tacitus, Germania (interpretiert, herausgegeben, übertragen, kommentiert und mit einer Bibliographie versehen von A.A. Lund), Wissenschaftliche Kommentare zu griechischen und lateini-schen Schriftstellern, Heidelberg.

Łowmiański H.

1963 Poczatki Polski. Z dziejów Stowian w I tysiacleciu n.e., v. I, Warszawa.

Madyda-Legutko R.

1996 Zróżnicowanie kulturowe polskiej strefy beskidzkiej $w$ okresie lateńskim $i$ rzymskim, Rozprawy Habilitacyjne nr 304(1), Kraków.

2004 Polskie Karpaty wokresie późnolateńskim i wokresie wptywów rzymskich. Uwagi dotyczqce zróżnicowania kulturowego, [in:] Okres lateński i rzymski w Karpatach polskich, J. Gancarski (ed.), p. 71-92.

Madyda-Legutko R., Pohorska-Kleja E., Rodzińska-Nowak J.

2009 Pakoszówka, Gde. und Kr. Sanok, Woiw. Podkarpackie, Fst. 1 (Siedlung aus der Römischen Kaiserzeit), Recherches Archéologiques Nouvelle Serie 1, p. 311-320.

Madyda-Legutko R., Rodzińska-Nowak J.

2009 Sytuacja kulturowa $w$ dorzeczu górnego Sanu $w$ okresie rzymskim $w$ świetle najnowszych badań, Rocznik Przemyski 45(2). Archeologia, p. 42-55.

Madyda-Legutko R., Rodzińska-Nowak J., Zagórska-Telega J.

2009 Prusiek, Fst. 25, Gde. und Kr. Sanok, Woiw. Podkarpackie-das erste Gräberfeld der Bevölkerung der Przeworsk Kultur in den polnischen Karpaten, Recherches Archéologiques Nouvelle Serie 1, p. 295-309.

Nosek S.

1961 Pèriode romaine, Inventaria Archaeologica Pologne, Fasc. VI: PL 35, WarszawaŁódź.

Nowakowski W.

2017 Okucie rogu do picia z Legionowa na tle znalezisk plastyki bukefalicznej $w$ Barbaricum (zarys problematyki $i$ kierunków planowanych poszukiwań badawczych), [in:] Relacje kultury przeworskiej i lateńskiej na Mazowszu. Wnioski $z$ badań cmentarzyska przeworskiego $w$ Legionowie CSP, W. Nowakowski, W. Orliński, M. Woińska (eds.), Legionowo-Warszawa, p. 119-134. 
Olędzki M.

2008 Czas przemian. Barbaricum między Battykiem a środkowym Dunajem $w$ dobie wojen markomańskich, Łódź.

2017 The Problems connected with the Identification and Localisation of the Suebian Tribe of Buri, Ephemeris Napocensis 27, p. 187-196.

Polaschek E.

1965 Ptolemaios als Geograph, [in:] Realenzyklopädie der classischen Altertumswissenschaft, Supplement-band 10, Stuttgart, col. 680-833.

Poleska P.

1996 Die latènezeitliche Siedlung in Kraków-Nowa Huta-Pleszów, [in:] Kontakte längs der Bernsteinstraße (zwischen Caput Adriae und den Ostseegebieten) in der Zeit um Christi Geburt, Z. Woźniak (ed.), Kraków, p. 211-242.

2005 The Celtic settlement microregion in the area near Kraków, [in:] Celts on the Margin. Studies in European Cultural Interaction 7th Century BC-1st Century AD Dedicated to Zenon Woźniak, H. Dobrzańska, V. Megaw, P. Poleska (eds.), Kraków, p. 187-194.

2006 Celtycki mikroregion osadniczy w rejonie podkrakowskim, Biblioteka Muzeum Archeologicznego w Krakowie II, Kraków.

Przybyła M.J.

2004 Nowe znaleziska kultury lateńskiej z obszaru Pogórza Rzeszowskiego, [in:] Okres lateński i rzymski w Karpatach polskich, J. Gancarski (ed.), p. 219-235.

Reichert H.

2003 Ptolemaeus, [in:] Reallexikon der Germanischen Altertums-kunde, 23, H. Beck, D. Geuenich, H. Steuer (eds.), Berlin-New York, p. 567-597.

Schmidt L.

1910 Geschichte der deutschen Stämme bis zum Ausgange der Völkerwanderung, Berlin.

1941 Geschichte der deutschen Stämme bis zum Ausgange der Völkerwanderung. Die Ostgermanen, München.

1942 Geschichte der Wandalen, Aufl. 2, München.

Stahl M.

1989 Zwischen Abgrenzung und Integration: Die Verträge der Kaiser Mark Aurel und Commodus mit den Völkern jenseits der Donau, Chiron, Mitteilungen der Kommission für Alte Geschichte und Epigraphik des Deutschen Archäologischen Instituts 19, p. 289-317.

Steche Th.

1937 Altgermanien im Erdkundebuch des Claudius Ptolemäus, Leipzig.

Strobel K.

1984 Untersuchungen zu den Dakerkriegen Trajans. Studien zur Geschichte des mittleren und unteren Donauraumes in der Hohen Kaiserzeit, Antiquitates 1, Abhandlungen zur alten Geschichte 33, Bonn.

Tausend K.

1997 Lugier - Vandilier - Vandalen, Tyche: Beiträge zur Alten Geschichte, Papyrologie und Epigraphik 12, p. 229-236.

Timpe D.

1992 Tacitus' Germania als religionsgeschichtliche Quelle, [in:] Germanische Religionsgeschichte. Quellen und Quellenprobleme, H. Beck, D. Ellmers, K. Schier (eds.), Ergänzungsbände zum Reallexikon der Germanischen Altertumskunde 5, Berlin-New York, p. 434-485. 
Vasmer M.

1958 Lugii und Mugilones, [in:] Sybaris. Festschrift Hans Krahe zum 60. Geburtstag am

7. Februar 1958 dargebracht von Freunden, Schülern und Kollegen, Wiesbaden, p. $189-194$

Vulpe R.

1976 Studia thracologica, Bucharest.

Wenskus R.

1977 Stammesbildung und Verfassung. Das Werden der frühmittelalterlichen gentes. 2. unveränderte Auflage, Köln-Wien.

Woźniak Z.

1990 Osada grupy tynieckiej a Podtężu, woj. krakowskie, Wrocław-Warszawa-Kraków.

2004 Rola Karpat w okresie lateńskim, [in:] Okres lateński i rzymski w Karpatach polskich, J. Gancarski (ed.), p. 43-70.

\section{Antike Schriftquellen}

Diо, $H R$

Dionis Cassii Cocceiani Historia Romana. Editionem primam curavit Ludovicus Dindorf recognovit Ioannes Melber, vol. I-III, Bibliotheca Teubneriana, Lipsiae MDCCCXC- MCMXXVIII.

PтоL., Geogr.

Claudii Ptolemaei Geographiae, ed. J. Fischer, Codex Urbinas Graecus 82, Codices Vaticanis Selecti, v. XVIII, Bibiotheca Apostolis Vaticana 1932.

SHA

Scriptores Historiae Augustae edidit E. Hohl, vol. I, Bibiotheca Teubneriana, Lipsiae 1965.

TAC., Ann.

Publius Cornelius Tacitus, Ab excessu divi Auguti (Annales), E. Koestermann (ed.), Bibiotheca Teubneriana, Lipsiae 1952.

TAC., Germ.

P. Cornelii Taciti libri qui supersunt, E. Koestermann [ed.], vol. II, fasc. 2: De origine et situ Germanorum (Germania), Bibiotheca Teubneriana, Lipsiae 1962.

Address of the Author

\section{Wojciech Nowakowski}

Institute of Archaeology

University of Warsaw

Krakowskie Przedmieście 26/28

00-927 Warszawa, Poland

samland@wp.pl

ORCID ID: 0000-0002-2040-7487 
